\title{
Natural Selection and Material Culture
}

\author{
Nathan Goodale $\cdot$ George T. Jones $\cdot$ Charlotte Beck
}

Published online: 26 July 2011

(C) Springer Science+Business Media, LLC 2011

\begin{abstract}
Natural selection is the basis of all evolutionary applications in biology as well as studies of cultural process in archaeology. Natural selection is important because it allows us the tools to talk not only about variation in biological systems but also material culture that are the byproducts of the human decision-making processes. In this paper, we provide a baseline of the concept of natural selection and explanatory application in evolutionary archaeology.
\end{abstract}

Keywords Natural selection · Fitness · Drift - Variation . Cultural transmission $\cdot$ Network analysis $\cdot$ Replicative success $\cdot$ Signaling

The central concept in Darwin's theory of evolution is that of natural selection, the mechanism by which biological populations change over time. Although Darwin understood natural selection on a general basis, defining it as the "preservation of favourable variations, and the rejection of injurious variations" (1859), he did not know how it actually operated because genetics was unknown at the time. He implied, however, that it was through reproduction that particular variations, or traits as we call them here, were or were not passed on to future generations. Today, the concept of natural selection is applied not only to biological variation but also to human behavior and the products of that behavior-material culture. Our purpose here is to explore the concept of natural selection and then to outline how it has been used in evolutionary archaeology to explain variation in material culture as a by-product of human behavior.

N. Goodale $(\bowtie) \cdot$ G. T. Jones $\cdot$ C. Beck

Hamilton College, Anthropology Department, Science Center, Clinton, NY 13323, USA

e-mail: ngoodale@hamilton.edu

\section{How Does Natural Selection Work?}

Most of us who have taken a course in biology will be familiar with the mantra that natural selection operates on the individual, but it is the population that evolves. Each individual has a unique genetic makeup (their genotype), which, in conjunction with the environment, determines the physical nature of that individual (their phenotype). It is the phenotype upon which selection operates, but it is the genetic information that is passed on from parent to child. Not all genetic information is evident in the phenotype, however. For example, a dominant trait will have phenotypic expression even if inherited from only one parent, but to have such expression, a recessive trait must be inherited from both. Thus, in the latter case, a trait that is recessive will not come under selection, whether it is beneficial or deleterious, because it is not expressed phenotypically; that is, it will not affect the fitness of the individual carrying it. An individual's fitness is determined by his or her success in producing offspring that, in turn, survive to produce offspring themselves. Those individuals that are most successful in producing offspring are the "most fit," and will, by definition, contribute the most genetic material to future generations. Therefore, from generation to generation, the genetic makeup of a population of interbreeding individuals will change; that is, the population evolves.

Even if genetic information is expressed in the phenotype, only some of these traits will be under selection. We can distinguish between those that are (functional) and those that are not (adaptively neutral). In general, the frequency of a beneficial trait that is under selection will increase steadily in the population toward some arbitrary value, while that of a neutral trait will drift randomly from generation to generation, eventually becoming fixed at $100 \%$ or disappearing altogether (Holland and Holland 
1990). Thus, the frequency of neutral traits in a population is due not to natural selection but to random processes, the primary of which is drift. Whether a trait is functional or neutral is context-specific; a trait that is neutral in one circumstance may come under selection in another.

Endler (1992:221) argues that three conditions must hold in order to argue that natural selection is operating:

The population has (a) variation among individuals in some attribute or trait (phenotypic variation); (b) a consistent relationship between that trait and mating ability, fertilizing ability, fertility, fecundity, and/or survivorship (fitness variation); and (c) a consistent relationship, for that trait, between parents and their offspring, which is at least partially independent of common environment effects (inheritance).

The crucial condition here is fitness variation (b). If conditions (a) and (c) are met but (b) is not, then any observed change is due to drift.

These conditions can be illustrated using a classic example. Male peacocks thoroughly perplexed Darwin because he saw no selective advantage for some males to bear such long trains (the long tails with "eyes" for which peacocks are so well known). The trains are heavy, make peacocks more susceptible to predators, and are expensive metabolically to produce. Since all male peacocks cannot provide the same amount of energy toward the growth of their train, there is phenotypic variation in peacock trains [Endler's condition (a)]. What we now know is that male peacocks with well-endowed trains tend to get more mates than those with more modest trains and also produce more fit offspring (Hale et al. 2009). Because a well-endowed train influences mating ability and signals survivorship [fitness variation, Endler's condition (b)], and offspring inherit the capacity to grow a train and use it as a signal of inheritance in whole or part from their parents [Endler's condition (c)], then natural selection can be said to have shaped this relationship. The peacock train is then, by definition, an adaptation. If condition (b) did not hold true, and a male peacock's train did not impact fitness (peacocks with less well-endowed trains found mates in the same frequency and fitness levels as those with well-endowed trains), then that character state (male peacock train) would not be subject to selection and therefore would be subject to drift.

\section{Natural Selection, Human Behavior, and Material Culture}

Although the impact of natural selection with regard to biological features is well understood, its influence on human behavior is a topic of controversy. The debate concerning the genetic basis of human behavior has raged for half a century, spawning the new field of sociobiology and, more recently, evolutionary psychology. While the level of genetic contribution to human behavior is debated, what is evident is that behavior is part of the human phenotype and thus susceptible to natural selection. Beginning in the 1970s, some researchers, archaeologists in particular, began to argue that not only is human behavior subject to natural selection, but also the products of that behavior, in particular, components of material culture (e.g., Dunnell 1978a, b). As humans have evolved, we have lost many of our biological mechanisms for protection and display, such as body hair, claws, and long canines, the functions of which are now performed by our technology. By extension, then, technology must contribute to our fitness, and thus the argument has been made by evolutionary archaeologists that the material products of behavior should be considered part of the human phenotype (e.g., Leonard and Jones 1987). Because natural selection operates on the phenotype, by definition, then, it operates on material culture.

From this perspective, Endler's argument concerning natural selection might be rephrased to include material culture: (a) that there is variation in the technology humans make and use, (b) that some variants affect fitness, and (c) that a form of inheritance occurs when we learn how to make and use technology (information is passed from the teacher to learner). Let's consider each of these in more detail using an archaeological example: the transition from the atlatl and dart technology to the bow and arrow in North America. We begin with some background on this transition.

Prior to about 9,000 years ago, the spear or javelin, propelled by hand, was replaced by a new propulsion system, the spear thrower, or atlatl (see, for example, Hughes 1998). The atlatl, which acts as an extension of the arm, significantly increased the distance a spear could be thrown (Fig. 1). This new technology comprised three basic components: the atlatl, the spear (termed a dart), and the stone projectile tip, or dart point. Archaeological examples illustrate that the morphologies of these components varied

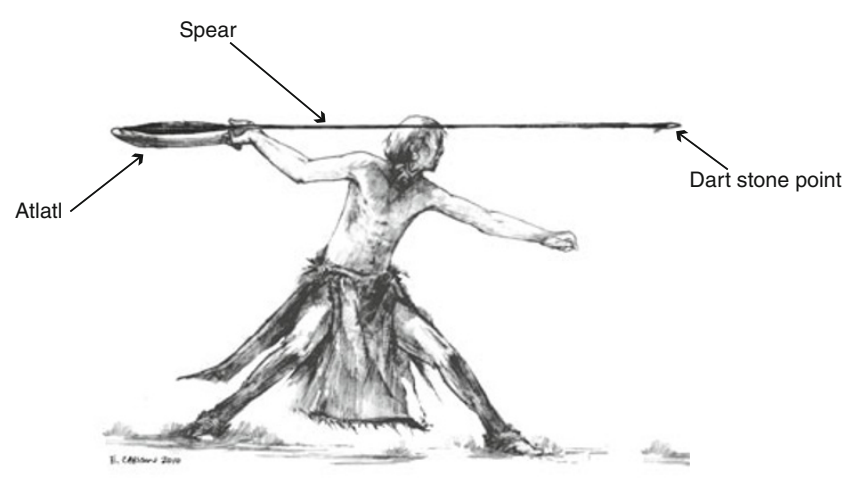

Fig. 1 The use of an atlatl and components of the technology 
across geographic regions as well as over the 7,000 years during which this technology was in use. About 2,000 years ago, the bow and arrow appeared, replacing the atlatl and dart almost entirely. Because this replacement was relatively rapid, it is believed that this new technology offered considerable advantage to hunters. So, how do we portray this transition within Endler's model as it has been recast for material culture? We begin with phenotypic variation.

\section{Phenotypic Variation}

For natural selection to operate, there must be several alternatives that can complete the same task. This variation may exist within or between technologies. For example, within the atlatl and dart technology, there are varied forms of atlatls, some comprising a single piece and others of several parts. Some incorporate weights for balance while others do not, some have handles, others grips, and so forth. The dart points vary in size, form, and raw material from which they are made.

At a more inclusive scale, the variation is between the two technologies, which differ in propulsive force, accuracy, and so forth. Selection here is operating on the totality of components comprising the technology rather than on variations within individual components. This is not to say that variation did not exist within components of the bow and arrow; it most certainly did, and changes in that technology after it was introduced (such as the bow size and form, arrow shaft form, point size and form, e.g., Fig. 2) probably resulted from the influence of natural selection. The advantages of the bow and arrow over the atlatl and dart, however, are undoubtedly what led to the replacement, which brings us to fitness variation.

\section{Fitness Variation}

As is the case for biological traits, cultural traits can be either functional or neutral, the latter designated as stylistic, and their status at any one time is context-specific (Dunnell 1978a, b). Also like functional biological traits, not all functional cultural traits enhance fitness. For example, some traits may be carried along from generation to generation because they "piggyback" onto one or more traits that are under strong, positive selection. The criterion of fitness variation requires only that some variants affect fitness. Our two propulsion technologies, on the whole, represent functional traits, although there are characteristics in the individual components of each that could be stylistic. But at least some of functional traits of these technologies affect fitness. Although it is difficult to point specifically to those that do so, we can examine what fitness advantages the bow and arrow may have conferred on its users over the atlatl and dart. First, the bow and arrow had the same
Fig. 2 Morphological distinction between Besant dart technology (left) and Avonlea arrow technology (right)
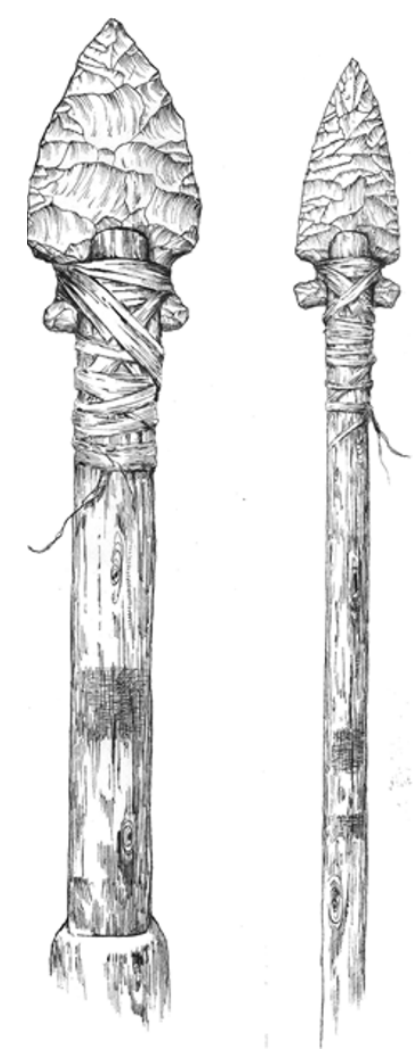

advantage over the atlatl and dart that the latter had over the javelin: greater propulsion distance. This would have allowed a hunter to launch farther away from his prey, lessening the possibility that he would have been discovered. In addition, it also had the advantages of greater missile speed (because of the greater force propelling the arrow) and greater precision and accuracy, in part due to the greater speed. These differences would have increased a hunter's efficiency, allowing the capture of more game.

\section{Inheritance}

This component has been one of the most contentious among those who reject the application of Darwinian principles to explain artifact variation; artifacts don't reproduce like biological organisms and thus cannot inherit anything. Evolutionary archaeologists argue, however, that the learning process, in which information is passed from one individual to another, what archaeologists call cultural transmission (Boyd and Richerson 1985), is analogous to biological inheritance. Cultural transmission is learned and can occur between parent and offspring (vertical transmission), between learners and relatives or other more experienced members of society (oblique transmission), or from peer-to-peer interaction (horizontal transmission). Cultural transmission can also vary by how much instruction is given in learning the craft (of say making projectile points) and/or differ in the extent to which there is a fitness 
benefit related to reproductive success; it could also relate to some potential prestige a person may obtain based on some behavioral variant. However, not all traits that are conveyed through cultural transmission are functional; stylistic traits are also conveyed in this manner.

Archaeologists have utilized the concept of cultural transmission on an intuitive basis for over a century in the form of "culture contact" and diffusion, but only through evolutionary archaeology has it been the focus of intense study. Similarity in form, especially for style, indicates some degree of contact and transmission, but this has been difficult to measure. Behavioral scientists do not agree upon what constitutes a "package" of transmitted cultural information; we have no direct unit equivalent to the gene. Further, we know that the packages are stored in different ways and reconstituted in complex ways of generating manifold opportunities for innovation and consequent selection, making the measurement of cultural transmission even more difficult. One recent approach in physics (based on statistical inference) has proved quite useful in graphing how similar/different one object is from another through network analysis (see Farrah et al. 2009). Network analysis reveals how a technological tradition, a set of rules or templates for properly making a particular item by members of a social group, is translated into a set of physical objects. Goodale et al. (2011) applied this approach to a group of projectile points (both dart and arrow points), from the northern Plains of North America. First, a set of morphometric measurements were taken from a set of landmarks on the points to describe their morphology, and then comparisons of each point to all others were made statistically, resulting in quantitative measures of similarity. The results are represented in a network diagram where each point is designated by a circle (or node, Fig. 3). Lines (degrees) connect nodes that are most similar in morphology. As we can see, the network is partitioned into two subgroups, which happen to correspond to cultural units (called types) that archaeologists previously have labeled Besant and Avonlea traditions of projectile point manufacture. The blue

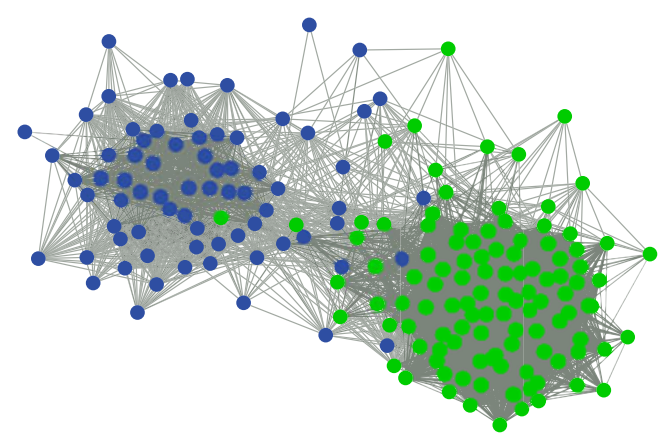

Fig. 3 The dart-to-arrow transition graphed by technology. Besant dart points are indicated with blue nodes and Avonlea arrow points are indicated with green nodes nodes represent points belonging to the Besant dart point tradition, while the green nodes correspond to the Avonlea arrow point tradition. These results indicate that the Besant and Avonlea point types are fairly distinct from one another, suggesting two sub-networks in a larger network of making projectile points. That is, there are different templates for making these two types of points and these templates were transmitted to members of the social group in which each is made (cladistical approaches have similarly been applied to identifying material cultural lineages, e.g., O'Brien and Lyman 2001).

Once natural selection is in operation on material culture, the question is, how do we judge fitness, which for biological organisms is measured by reproductive success? As stated above, components of material culture do not genetically reproduce so logically they cannot have reproductive success. They may enhance the reproductive success of their users, although this can be difficult for archaeologists to measure directly. But in exactly the same way, it is difficult for paleontologists to measure the fitness benefits of morphological variation. Evolutionary archaeologists instead view material goods manufactured by humans to have replicative success or differential persistence through time and across space (Leonard and Jones 1987). What this means is that given a set of alternative forms, some will be chosen by users more often than others, and those chosen forms will be made known to other users through vertical, horizontal, or oblique transmission at the expense of the less desired forms. Replicative success is a useful concept because the relative abundance of technological forms, the amount of variation within a technology, and the causes for that variation allow us to operationalize natural selection in archaeology.

Summarizing this discussion, it is evident that the transition from atlatl and dart to bow and arrow fulfills all three of Endler's criteria: (a) there is variation between these two technologies in the maximum effective distance between user and target, speed of the launched missile, and the precision and accuracy in hitting the target; (b) there is fitness variation between these two technologies, such as the proximity of the user to the target and the degree of success in the user hitting the target; and (c) there is cultural transmission resulting in substantial replicative success, indicated by the speed and degree of replacement.

Recall, however, that in Endler's model, if fitness variation (that is, traits that enhance fitness) is not present, then the change in a particular trait will be due to drift. Thus, both functional and stylistic traits can have replicative success, the former due to natural selection and the latter due to drift. In most cases, artifacts comprised both functional and stylistic (adaptively neutral) traits. However, determining which traits are functional and which are stylistic can be a difficult task. One means that we have 
of determining function from style is through experimental archaeology where replication and use of a technology is analyzed through practice. In experimental archaeology, an analyst may replicate projectile points with the same dimensions as dart points and arrow points and then test their relative efficiencies in performance. In the case of a pottery vessel, we may use instruments to analyze the thermal properties in cooking and their performance. However, a more nuanced approach in terms of looking at the time depth in the archaeological record is to examine how differential attributes behave over time to identify them as either stylistic or functional.

In general, functional traits tend to spread rapidly once they are invented or introduced if they offer an immediate advantage to the recipients. As our example shows, the bow and arrow conveyed several advantages to its users over the atlatl and dart and thus was adopted relatively quickly. On the other hand, pottery, which was present among semisedentary horticulturalists in the southwestern U.S. more than 1,500 years ago, was only adopted very late in prehistory by the mobile hunter-gatherers in the adjacent Great Basin, and even then was used sparingly. Pottery provides a much better cooking vessel than basketry and boiling stones, but it is heavy and it breaks, two negative factors for people who move their camps on a regular basis.

A functional trait will remain in use until something better comes along, which, in some cases, can be a long period of time. Functional traits, however, may come and go over time, as needs change. A classic functional example is the stone endscraper, used for scraping animal hides. The earliest of these tools appear hundreds of thousands of years ago, and although their frequencies have fluctuated through time (making their temporal frequency distributions multimodal), they persisted in roughly the same form until the appearance of metal tools. In contrast, the temporal frequency distributions of stylistic traits tend to be unimodal through time. After inception, they gain popularity, rise to a peak, then fade and eventually disappear. Styles rarely return in the same form. This unimodal distribution through time and the fact that they rarely return make stylistic traits especially useful for chronology. Once a time span can be identified with the unimodal distribution of a particular style, the presence of this style at different locations indicates the temporal period during which it was deposited.

From this discussion, it may seem fairly simple to distinguish functional from stylistic traits, but this process is not so straightforward. Where the temporal distribution of functional traits is more often multimodal, it can also be unimodal, mimicking that of a stylistic trait. On the other hand, a stylistic trait can spread relatively quickly where the population is highly mobile and there are few social boundaries, thus mimicking a functional trait under strong selection. To complicate the situation further, the status of a trait is context-specific and thus can change as conditions and selection pressures change. In some cases, this distinction is not an important one, such as the technological replacement example discussed earlier where the advantages conferred by one variant over the other are easily identified. In others, however, the distinction may be necessary because the variants compared may be quite similar, making it difficult to figure out which traits enhance fitness. Therefore, multiple forms of evidence are necessary in an attempt to distinguish one from the other (e.g., performance characteristics, analogous changes in comparable contexts, temporal and spatial patterns).

\section{Barbie, Signaling, and Natural Selection}

Trained as archaeologists, we are quite used to thinking about questions like "why did arrow points replace dart points?" In such cases, performance-based studies or engineering perspectives seem to provide reasonable answers. To the degree that arrow points brought greater efficiency to food procurement, they would have enhanced the survival of their users and thus constituted a technological adaptation. Further, we would have to conclude that it was a combination of features, a technological system, which included the physical media, the mechanical actions of the archer, and the knowledge required to effectively use and reproduce the components of the system, and not just the projectile tip, that was under positive selection and increased fitness. None of this seems too far-fetched despite the fact that we probably cannot demonstrate that early adopters of this technology achieved enhanced reproductive success relative to those that may have held on to the alternative technology. We also suspect, but cannot easily demonstrate, that the speed with which the shift from darts to arrows occurred probably was accentuated by some additional process, like conformist or prestige-based cultural transmission (there is an added prestige incentive to have replicative success above survival fitness). And yet there are many other changes in material culture for which appeals to improvements in design and increased efficiency do not appear to be satisfactory. We do not need to look to an esoteric example from prehistory to make this point; there are plenty of examples in the material culture with which we surround ourselves. Take, for example, Converse sneakers. Converse sneakers were extremely popular in the $1950 \mathrm{~s}$, worn in basketball and for leisure. While they continued to be manufactured and used in the sport during the ensuing decades, companies like Nike and Reebok started to make large impacts in basketball shoe sales and their technological advancements rendered Converse obsolete. The result was a loss 
of popularity for Converse on the basketball court during the 1980s and 1990s among basketball players and the consumers (fans) of basketball. However, Converse shoes again became popular during the 1990s within a different social movement: Seattle-based grunge rock led by the late but great Kurt Cobain, lead singer and guitarist of Nirvana. This example illustrates that the selection matrix often is not unidimensional. You could say that for some period, Converse and Nike shoes competed, but later moved into separate niches. This is a feature probably of most modern, consumer-oriented technology. But if we are honest, the factors influencing the cultural selection of technology have been complex for a very long time, certainly at least since our ancestors began to embellish tools, living structures, and the landscape with symbols.

In "Archaeology as Anthropology," a paper that ushered in "processual archaeology," Lewis Binford (1962) reminded archaeologists that artifacts function not just in the sense of task performance but in social and ideological contexts as well. Archaeologists' efforts to explain the social and ideological roles of artifacts have drawn largely from anthropological theory and have turned on a functional theme attributable to Emile Durkheim. This view can be stated simply as a question: how do the social and ideological roles of artifacts act to achieve and maintain social integration? Recently, some archaeologists have tried to come to grips with the social and ideological roles of artifacts from an expressly evolutionary perspective. The key feature of this perspective is the recognition that many artifacts exhibit far more effort in their production than is necessary for the strictly functional utility of the artifact. In many instances, those artifacts are so costly to produce that the expenditure on them is excessive, even wasteful, fitting Veblen's (1899) notion of "conspicuous consumption." From a Darwinian standpoint, this effort seems counterintuitive since it appears to come at the expense of fitness. But like the peacock, whose elaborate and costly plumage pays off with successful attraction of mates, elaborated artifacts may likewise advertise qualities of the bearer and engender responses on the part of the receiver that are to the benefit of the bearer. This theoretical perspective has been effectively conveyed in Neiman's analysis of Mayan stone monuments (Neiman 1997). We offer a more mundane example below to illustrate the complex selection matrix that influences the persistence of much material culture. For this example, we turn to Barbie.

Barbie is a doll, of course, a piece of plastic, a parody of human physique or a model of physical perfection depending on your perspective, and she is coveted by many young (and some older) females, mostly. She is enormously popular; she, no doubt, has great replicative success. But why would she interest an archaeologist? For one thing, her success, combined with the much more variable success of other dolls representing Barbie's siblings, neighbors, girlfriends, and boyfriends, illustrates very nicely the temporal patterns of artificial clades (a group of individuals such as organisms or projectile points that are related by descent). Some members of Barbie's clade, like Barbie herself, are long-lived and reach high frequencies and wide distributions, while others persisted effectively just for an instant. We needn't worry why this is. It is enough to show that these qualities of persistence and frequency match those of classes of artifacts that archaeologists call historical types. In this sense, Barbie and her brethren are like design motifs on pottery or the shapes of battle axes. Barbie's chronological behavior is confirmation that there is really no disjunction between the archaeological past and the present; some kinds of material cultural work the same way.

Still, there is more than a time chart of shifting popularity to provoke an archaeologist's interest in Barbie. Perhaps you've already made the connection with the earlier example in this paper of the peacock's elaborate plumage. Barbie can certainly be dressed in elaborate plumage, and that point begins to touch on why this may be an apt comparison. To explain why the male peacock makes such sizeable investments, biologists mount arguments as to the effects of sexual selection and costly signaling to account for peacock fitness, which is advertised by their elaborate plumage. A biologist might say that the male peacock uses its plumage not only to attract the female but also to convey something of its greater vigor relative to other peacocks. If that proves to be an honest signal, that is the peacock's plumage positively correlates with other advantageous characteristics, wise females selecting those males as mates will have greater reproductive success. If the behavior is passed on to offspring whereby female peacocks make such decisions based on a proxy like plumage, there will be positive selection for more elaborate plumage.

Like peacocks, humans are very sensitive to signals. To make this point, we need look no further than the multiplicity of signals that reach us in language. All of our senses, aural, visual, tactile, olfactory, receive signals. And because so much of our behavior is manifest in material culture - our phenotype is so enhanced by material culture-we produce and receive a staggering array of material signals. Like linguistic signs, we use material signals for their referential value, that is, to denote objects and activities, and indexically, wherein signals are associated with individuals or social groups, marking them as distinct from other individuals or groups. Barbie offers considerable opportunity to construct signals. She comes in a wide variety forms - different ethnicities, occupations, leisure choices, not to mention hair colors, the first trait to vary. The choice of a variant or the manipulation of traits can convey some form of personal identity of the child. 
However, the replicative success of Barbie's variants will denote waxing and waning of popular Barbie styles through time, and this may in turn be explained by the signaling of the most successful (admired, inventive, copied) Barbie users.

Barbie play is a training ground for signal construction. This behavior appears early. It is practiced and enhanced by imitation (and other modes of transmission) and is reinforced by peers, parents, and media, and through play is adapted to many other types of material culture. The signals conveyed may reproduce normative cultural content and reinforce affinities between playmates (promote social solidarity), or they can be meant to show the distinctiveness of the user and even convey some measure of the user's dominance over playmates. Dominance might come from certain girls having the family fitness levels (e.g., income) that can purchase a Barbie (or multiple Barbies and many accessories) rather some other less expensive doll. The same holds true for other items of material culture whether the expense is monetary or in other instances may be expensive in terms of energy to get the materials to produce (such as an exotic rock that is not immediately accessible to make a projectile point).

This tangential linkage to signaling can be put on firmer theoretical ground. Barbie is used in play. Play is a part of juvenile behavior in many species, particularly those with extended periods of development and among whom surplus resources are available. According to Fagan (1974:850), play is "active, oriented behavior whose structure is highly variable, which apparently lacks immediate purpose, and which is often accompanied by specific signal patterns." From the standpoint of the function of play, its apparent absence of purpose calls for explanation. Groos (1901) and many later workers suggest that juvenile play is important for shaping later development; that is, the benefits of play are deferred into adulthood. As Pellegrini et al. (2007:263) put it, immature organisms use play "to sample their environment in order to develop adaptive behaviors" and to respond "to environmental novelty [which] may influence subsequent evolutionary processes." They go on to say that relative to other learning strategies, as for instance between parent and child, which tend to transmit existing practices, play is associated with greater behavioral innovation and more varied symbolic production. Thus, Pellegrini et al. (2007) conclude that play may have both immediate as well as delayed benefits. With sufficient resources available to juveniles, play is also relatively low-cost and free of risk. Moreover, as resource availability rises (especially time), the rates of play increase. Thus, we might expect across archaeological contexts that the frequency and elaboration of artifacts related to play will show variation corresponding to the level of excess resource control and social status difference in society.
Truth be told, we do not spend a great deal of time worrying over what sort of information might be conveyed through signaling using Barbie. Our example, however, serves as a reminder that material culture is imbued with meaning. As archaeologists, we know that little of that meaning may be recovered from artifacts despite our best efforts in analysis. Still, though successes in translation may be small, we know that the meaningful content of artifacts often account for peculiar distributions and frequencies that otherwise cannot be explained by the working utility (function) of objects.

\section{Conclusions}

In this paper, we have argued that natural selection operates not only on biological variation but also on behavior and its by-products. In doing this, we have attempted to emphasize that behavioral variation and fitness have a necessary relationship. In recent years, the circumstances under which functional traits are subject to fitness variation and thus to evolution under natural selection has been questioned (e.g., Bamforth 2002; Eldredge 2009). That some technological variants have implications for the reproductive success of their users, however, seems self-evident. If not, why have human population sizes generally increased in parallel with technological innovation over the course of human history? Still, many archaeologists object to the notion that artifacts have a direct influence on fitness, presumably because such a relationship fails to account for human decision making as part of the algorithm relating tool performance to greater reproductive success. We don't deny the influence of decision making, but we note it is the variation among artifacts that very often yields efficiency differences and these, in turn, influence energy capture, a critical variable in achieving different levels of reproductive success. In point of fact, the relation between the hard parts - the artifactsand the soft parts - variable strategies and techniques for using technology - in what proportion each contributes to the efficiency of tool-using tasks is complex. But to deny that there is a relation between technology and fitness seems as wrong-headed as to deny that a shift in the basic structure of a dinosaur leg did not influence its speed, its efficiency in energy capture, and its fitness. Neither the archaeologist nor the paleontologist can demonstrate conclusively that reproductive success was influenced by respective shifts in technology or morphology. What we can do is construct plausible arguments built on performance or biomechanical criteria.

The evolutionary success of humankind owes as much to the extended phenotype and the physical media, routines, and knowledge comprising our technologies as to changes in our biology. Some would argue that it is precisely the 
complexity of our extended phenotype and the modes of transmission it embodies that distinguish it and make evolutionary theory inadequate or even inappropriate for accounting for cultural change over time. We disagree and conclude that it is the only theoretical system up to the task of explaining artifact variation and its role in the successes and failures over the long span of human history (also see Shott 2011). After all, we are not the only organism that has a complex behavioral repertoire, nor the only species that makes use of tools. But we acknowledge as well that simplistic attributions to reproductive success will not explain what is shared by such a variety of physical items as dart points, sneakers, or Barbie. As the Barbie example illustrates, what humans can contribute to these artifacts goes beyond strictly technological performance in a narrow task arena and many include very complex symbolic dimensions as well, whose exact meaning may be beyond our ability to identify. This is a difficult chasm to bridge, especially in those cases where the archaeological record is comprised primarily of stone artifacts. Although, due to the time depth that natural selection can take into account, it seems ideal for addressing questions about the material culture that represents human behavior in the archaeological record, we must somehow use the present without biasing our view of the past.

Acknowledgments We would like to thank Anna Prentiss and Niles Eldredge for inviting us to contribute to this special edition of Evolution: Education and Outreach. Thank you to Eric Carlson who illustrated Figs. 1 and 2. Figure 2 is used with permission of Dr. Douglas Macdonald, University of Montana. Thanks also to Lisa Fontes and Brian Connolly for their contribution to the production of Fig. 3. Thanks also to Alissa Nauman for her support and conversation around this topic. Any errors or flaws in logical thought are completely our own.

\section{References}

Bamforth DB. Evidence and metaphor in evolutionary archaeology. Am Antiq. 2002;67:435-52.

Binford LR. Archaeology as anthropology. Am Antiq. 1962;215-225.

Boyd R, Richerson PJ. Culture and evolutionary process. Chicago: University of Chicago Press; 1985.
Darwin C. On the origin of species by means of natural selection, or the preservation of favoured races in the struggle for life. London: John Murray; 1859.

Dunnell RC. Archaeological potential of anthropological and scientific models of function. In: Dunnell RC, Hall ES, editors. Archaeological essays in honor of Irving B. Rouse. The Hague: Mouton; 1978a. p. 41-73.

Dunnell RC. Style and function: a fundamental dichotomy. Am Antiq. 1978b;43:192-202.

Eldredge N. Material culture macroevolution. In: Prentiss AM, Kuijt I, Chatters JC, editors. Macroevolution in human prehistory: evolutionary theory and processual archaeology. London: Springer; 2009. p. 297-316.

Endler JA. Natural selection: current uses. In: Keller EF, Lloyd EA, editors. Keywords in evolutionary biology. Cambridge: Harvard University Press; 1992. p. 220-4.

Fagan R. Selective and evolutionary aspects of animal play. Am Nat. 1974;108:850-8.

Farrah D, Connolly B, Connolly N, Spoon HWW, Oliver S, Prosper $\mathrm{HB}$, et al. An evolutionary paradigm for dusty active galaxies at low redshift. Astrophys J. 2009;700:395-416.

Goodale N, Andrefsky W Jr, Osterhoudt C, Cueni L, Kuijt I. Cultural transmission and the production of material goods: evolutionary pattern and measuring morphology. In: Goodale N, Andrefsky Jr., editors. Lithic technological systems: stone, human behavior, evolution. Cambridge University Press; 2011 (in press).

Groos K. The play of man. London: Heinemann; 1901.

Hale ML, Verduijn MH, MØller AP, Wolff K, Petrie M. Is the peacock's train an honest signal of genetic quality at the major histocompatibility complex? J Evol Biol. 2009;22:1284-94.

Holland MJ, Holland TD. Variation, selection, and the archeological record. In: MB Schiffer, editors. Archaeological Method and Theory, Vol. 2. Tucson: University of Arizona Press; 1990. p. 31-79

Hughes SS. Getting the point: evolutionary change in prehistoric weaponry. J Archaeolog Method Theory. 1998;5:345-408.

Leonard RD, Jones GT. Elements of an inclusive evolutionary model for archaeology. J Anthropol Archaeol. 1987;6:199-219.

Neiman FD. Conspicuous consumption as wasteful advertising: a Darwinian perspective on spatial patterns in Classic Maya terminal monument dates. In: M Barton and Clark GA, editors. Rediscovering Darwin: evolutionary theory and archaeological explanation. Archaeological Papers of the American Anthropological Association No. 7; 1997. p. 267-90.

O’Brien MJ, Lyman RL. Cladistics is useful for reconstructing archaeological phylogenies: Palaeoindian points from the southeastern United States. J Archaeol Sci. 2001;28:1115-36.

Pellegrini AD, Dupuis D, Smith PK. Play in evolution and development. Dev Rev. 2007;27:261-76.

Shott MJ. Theory in archaeology. In: Goodale N, Andrefsky Jr W, editors. Lithic technological systems: stone, human behavior, evolution. Cambridge: Cambridge University Press; 2011 (in press).

Veblen T. The theory of the leisure class. New York: Macmillan; 1899. 